\title{
Pattern and process in a narrow hybrid zone
}

\author{
Richard G. Harrison
}

Department of Biology, Yale University, PO Box 6666, New Haven, CT 06511, U.S.A.

This paper examines variation in morphology and allozymes in a hybrid zone between two closely related eastern North American species of field cricket (genus Gryllus). I show that patterns of variation across the zone do not conform to a simple model of monotonic clinal variation. In fact, the hybrid zone is a mosaic of populations. Pockets of "pure" parental forms are found within the hybrid zone, and striking reversals in mean character index score occur along transects across the zone. Treating hybrid zones as mosaics has important consequences for thinking about the dynamics of such zones.

Patterns of variation in morphology and allozymes are not concordant across the hybrid zone. Rather, there is strong evidence for differential and asymmetric introgression, with morphological integrity maintained despite considerable introgression of alleles at allozyme loci. Species boundaries must be thought of as semipermeable, the permeability varying with ihe genetic marker used.

I also show that there is strong positive assortative mating at one site within the hybrid zone and that assortative mating persists despite introgression at allozyme loci. Habitat isolation and behavioural differences may both affect the extent of assortative mating.

\section{INTRODUCTION}

The origin, current dynamics and eventual fate of narrow hybrid zones remain subjects of considerable debate among evolutionary biologists. Hybrid zone origins have traditionally been discussed in the context of the dichotomy between primary intergradation and secondary contact (Mayr, 1942). Because these processes can produce identical patterns of variation, it is difficult, if not impossible to distinguish between them (Endler, 1977; 1983; but see Thorpe, 1984). Consequently, recent attention has focused on the dynamics of hybrid zones and the processes that contribute to their maintenance (e.g., see Endler, 1977; Moore, 1977; Barton and Hewitt, 1981).

Once formed (by either process mentioned above), hybrid zones may be thought of as stable or transient. Stability is usually viewed as the result of a balance between gene flow and selection (Slatkin, 1973; 1975; Endler, 1977; Barton, 1979a, b; Barton and Hewitt, 1981) or as a result of selection in favour of hybrids within an ecotone (Moore, 1977). A balance between gene flow and selection may involve: (a) selection against hybrids (independent of the environment) or (b) a switch in the selective values of genotypes along an environmental gradient. Direct evidence for the long-term stability of hybrid zones is generally not available, but on the basis of indirect evidence, it has been suggested that some zones may have persisted for thousands of years (Short, 1972; Jackson, 1973; Hall and Selander, 1973; Hunt and Selander, 1973).

Hybrid zones may represent transient phenomena. There are three commonly described scenarios for the eventual fate of a transient hybrid zone: (a) speciation via "reinforcement" (the evolution of pre-mating barriers to gene exchange in response to selection against hybrids); (b) fusion of the two parental types; (c) extinction of one or the other parental form (see Mayr, 1942; 1963; Wilson, 1965; Remington, 1968; Crosby, 1970; Paterson, 1978; Templeton, 1981). Although reinforcement is central to a major class of allopatric speciation models (Remington, 1968; Dobzhansky, 1970; Ayala et al., 1974), both theoretical arguments against it (Moore, 1957; Bigelow, 1965; Paterson, 1978; 1982) and the dearth of evidence for the predicted reproductive character 
displacement (Walker, 1974; West-Eberhard, 1983) suggest that reinforcement may not be a common evolutionary pathway. In fact, premating barriers to gene exchange may frequently arise outside the zone of contact-a fortuitous result of divergence, not a direct response to selection against hybrids.

To understand the current dynamics of hybrid zones and to make reasonable predictions about their eventual fate, it is essential to accumulate detailed information on both pattern and process within and adjacent to the zones. In previous papers (Harrison and Arnold, 1982; Harrison, 1983 ; 1985) I have documented the existence of an extensive hybrid zone between two eastern North American species of field cricket (genus Gryllus) and characterised a number of barriers to gene exchange between these species. Here I focus on a restricted segment of the hybrid zone, documenting patterns of variation on a finer scale than in the earlier work and comparing patterns of introgression for morphological and allozyme markers. In addition, I show that there is strong positive assortative mating within the hybrid zone, despite substantial introgression of alleles at loci coding for soluble enzymes.

The hybrid zone between Gryllus pennsylvanicus and Gryllus firmus extends from Virginia to Connecticut, and over much of its length it follows the eastern slopes of the Blue Ridge and Appalachian Mountains (Harrison and Arnold, 1982). G. firmus is a cricket of coastal and lowland areas from Florida to New England. G. pennsylvanicus is found in inland situations in the northeastern United States and extends south in the mountains to northern Georgia (Alexander, 1968; Harrison and Arnold, 1982; Harrison, unpublished). Both species overwinter in the egg stage and adults appear in late summer and early fall. The two cricket species differ in a number of morphological characters, but none of these alone is perfectly diagnostic. Among 25 loci assayed by standard allozyme techniques, none show fixed allelic differences, and the Nei (1972) interspecific genetic distance is only $0.02-0.03$ (Harrison, 1979 , unpublished). However, differences in allele frequencies at three loci (Esterase, Peptidase-1, Peptidase-3) are sufficiently great that alleles at these loci provide useful genetic markers. Crosses between $G$. firmus males and $G$. pennsylvanicus females give rise to viable and fertile offspring, whereas the reciprocal cross consistently fails to produce offspring (Harrison, 1983). This asymmetric outcome is not dependent on the locality from which the parents are obtained. Despite this partial post-mating barrier, individuals of mixed ancestry should be found in the field unless premating barriers are effective in preventing gene exchange. On the Blue Ridge in Virginia, temporal isolation of adults appears to be a significant (but not complete) pre-mating barrier. However, in Connecticut adults of the two species appear synchronously (Harrison, 1985).

\section{MATERIALS AND METHODS}

Crickets from 25 localities in Connecticut were used in this study (table 1, fig. 1). They represent 35 collections made in the late summer/early fall of the years 1980-1983. All crickets were frozen as adults (either immediately upon collection or after rearing in the laboratory from late instar nymphal stages) and stored at $-70^{\circ}$. Prior to homogenising head and thorax for electrophoresis, the tegmina (modified forewings) were removed from males and measurements made of body length $(\mathrm{BL})$, hind wing length (HWL), and ovipositor length (OL). Tegmina colour for each male (TEG) was determined by comparison with a standard series of tegmina (see Harrison and Arnold 1982). Horizon-

Table 1 Sample localities used in this study. The letter designation is used throughout the paper to refer to populations

\begin{tabular}{llll}
\hline & & & COLLEC \\
SAMPLE & TOWN & HABITAT & TIONS \\
\hline A & Bethany & roadside & 1980,1982 \\
B & Bethany & roadside & 1982 \\
C & Cheshire & roadside & 1981,1982 \\
D & Durham & old field & 1982 \\
E* & Guilford & beach/marsh & 1981,1983 \\
F & Guilford & old field & 1982 \\
G & Guilford & power line & 1982,1983 \\
H & Guilford & roadside & 1982,1983 \\
I & Haddam & grassy field & 1981 \\
J* & Hamden & roadside & 1980,1981 \\
K & Middlefield & grassy field & 1982 \\
L & Milford & beach & 1983 \\
M & Madison & beach & 1981 \\
N & Branford & roadside & 1982 \\
O & North Haven & roadside & 1980,1981 \\
P & Orange & roadside & 1982 \\
Q & Orange & roadside & 1982 \\
R & Seymour & roadside & 1982 \\
S & Sharon & pasture & 1981,1983 \\
T & Southington & roadside & 1981,1982 \\
U & Thomaston & roadside & 1982 \\
V & Wallingford & roadside & 1982 \\
W & Westport & dump & 1982 \\
X & Woodbridge & roadside & 1980 \\
$\mathrm{Y}^{*}$ & Waterbury & roadside & 1981 \\
\hline & & & \\
\hline & & &
\end{tabular}

* These populations were also sampled by Harrison and Arnold (1982). $E=G U 2, J=H N 2, S=S H 1, Y=W Y$. 


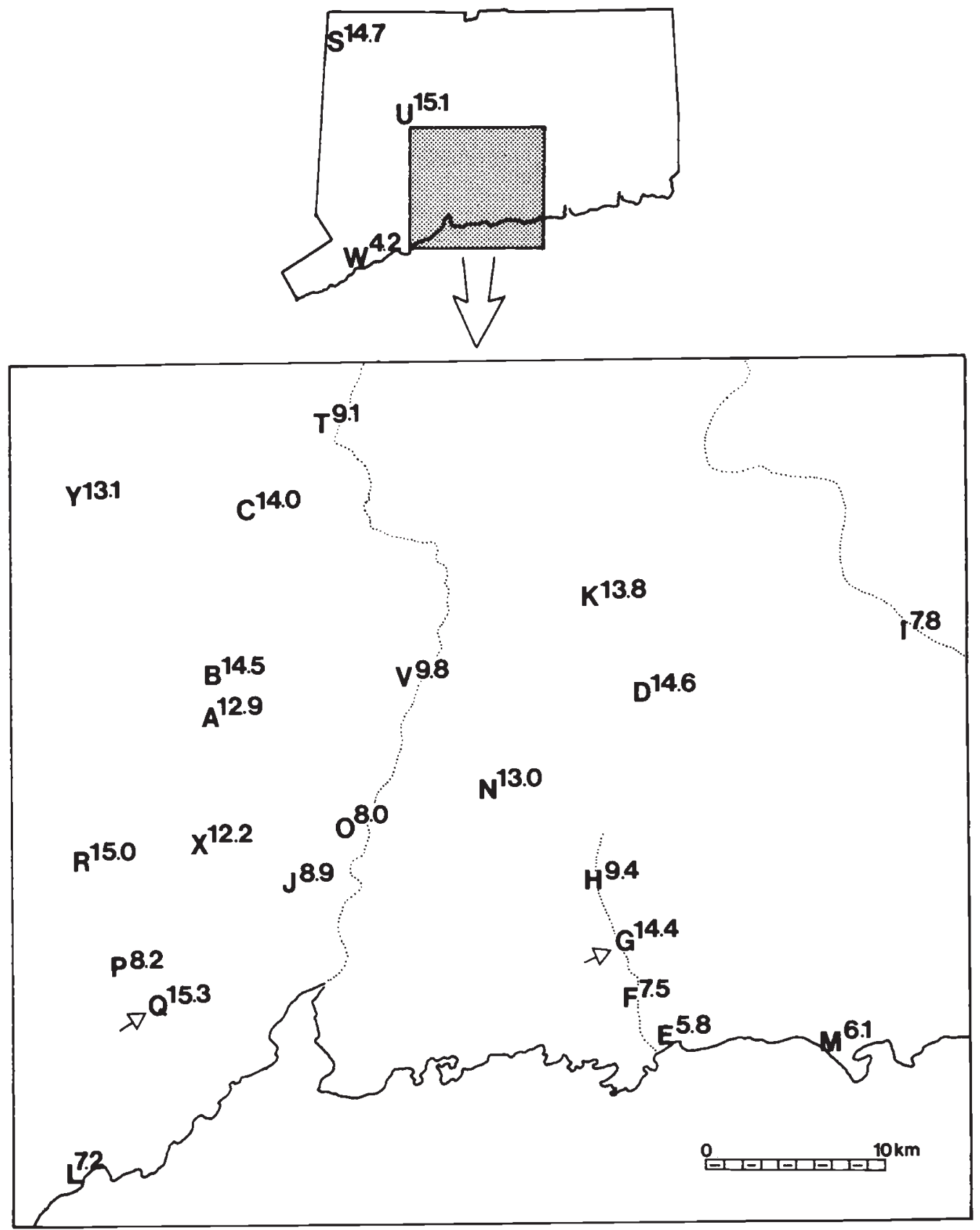

Figure 1 Map showing the location of the 25 collecting sites together with the mean character index score $(\bar{I})$ for crickets collected from those sites. The dotted lines are rivers. The arrows indicate pockets of "pure" $G$. pennsylvanicus within the hybrid zone.

tal starch gel electrophoretic techniques were as described previously (Harrison, 1979). In this paper I only report allele frequencies for a single Esterase locus (Est) and for two Peptidase loci (Pep-1, Pep-3). These three loci provide the most useful markers for distinguishing populations/species.
To characterise each field collected cricket, I constructed a character index score (I), based on morphological characteristics (TEG, HWL for males, OL, HWL for females) and genotype at each of the three allozyme loci. This index provides a convenient and easy-to-interpret summary of the characteristics of each individual cricket. A scoring 
system (table 2) was devised using information on morphology and allele distributions in populations considered to be "pure" $G$. firmus and "pure" $G$. pennsylvanicus (see Harrison and Arnold, 1982). Thus a female with $\mathrm{HWLF}=7 \cdot 0, \mathrm{OL}=18.5$ and genotypes $E s t^{-10 /-10}, P e p-1^{110 / 100}, P e p-3^{120 / 100}$ would have $I=2+1+0+2+1=6$.

Table 2 Method of calculating character index scores $(I)$ for individual field-collected crickets. Low scores $=$ " $G$. firmus", high scores = "G. pennsylvanicus".

(i) Assignment of scores for morphological characters

\begin{tabular}{|c|c|c|c|c|}
\hline SCORE & HWLM & TEG & HWLF & OL \\
\hline 0 & $>8 \cdot 0$ & $\leq 3$ & $>8 \cdot 5$ & $>19 \cdot 0$ \\
\hline 1 & $>7 \cdot 0, \leq 8 \cdot 0$ & $3 \cdot 5$ & $>7 \cdot 5, \leq 8 \cdot 5$ & $>17 \cdot 5, \leq 19 \cdot 0$ \\
\hline 2 & $>6.0, \leq 7 \cdot 0$ & $4 \cdot 0$ & $>6 \cdot 5, \leq 7 \cdot 5$ & $>16 \cdot 0, \leq 17 \cdot 5$ \\
\hline 3 & $>4 \cdot 5, \leq 6 \cdot 0$ & $4 \cdot 5$ & $>5 \cdot 0, \leq 6 \cdot 5$ & $>14 \cdot 0, \leq 16 \cdot 0$ \\
\hline 4 & $\leq 4 \cdot 5$ & $5 \cdot 0$ & $\leq 5 \cdot 0$ & $\leq 14 \cdot 0$ \\
\hline
\end{tabular}

(ii) Assignment of scores for alleles at Est, Pep-1 and Pep-3

\begin{tabular}{llll} 
SCORE & Est & Pep-1 & Pep-3 \\
\hline 0 & -10 & 100 & 120,70 \\
1 & $100,30,-40$ & 90,120 & 100 \\
2 & $70,60,35$ & 110 & -
\end{tabular}

(iii) Calculation of character index scores

$$
\begin{gathered}
I_{\text {male }}=I_{\mathrm{HWLM}}+I_{\mathrm{TEG}}+I_{E s t}+I_{P e p-1}+I_{P e p-3} \\
I_{\text {female }}=I_{\mathrm{HWLF}}+I_{\mathrm{OL}}+I_{E s t}+I_{P e p-1}+I_{P e p-3}
\end{gathered}
$$

Other easily measured morphological characters that have been used for describing field crickets (body length, tegmina length, femur length, pronotal length or width) are strongly correlated with HWL. All represent "size" variables, and only one of these characters was used in this analysis. The number of teeth on the stridulatory file is an important character for distinguishing Florida $G$. firmus from other species (Nickle and Walker, 1974). However data in Harrison and Arnold (1982) indicate that this character is not particularly useful for distinguishing the two taxa in Connecticut.

The character index score for each individual can be broken down into two components, $I_{e}$ (based only on enzyme genotypes) and $I_{m}$ (based on morphology alone). A population can then be characterised by its mean character index scores, $\bar{I}, \bar{I}_{e}$, and $\bar{I}_{m}$. Although the morphological characters used in constructing $I$ differ between the sexes, only in one population (E) was there a significant difference between the distributions of $I$ for males and females (for population $E, p<0.01$, for all other populations $p>0 \cdot 1$, t-test for differences between two means). Therefore, the values presented here are for both sexes combined.

To assay levels of assortative mating in the hybrid zone, I collected a large sample of fieldinseminated females from a population with a high variance in character index score $(\mathrm{J})$. Each female was provided with an oviposition dish (petri dish filled with moist sand-soil mixture) and allowed to oviposit for 7-10 days, after which the female was frozen. Eggs were treated as in previous studies (Harrison, 1983; 1985) and the resulting offspring of each female were frozen as 1st-3rd instar nymphs. From Pep-3 genotypes of mother and offspring, I estimated the frequency of the Pep- $3^{100}$ allele in the population of sperm contributed by the male parent(s). Because females are often multiply-inseminated, I could not unambiguously identify the Pep-3 genotype(s) of the male(s) that sired the female's offspring.

\section{RESULTS}

\section{Patterns of variation within and adjacent to the hybrid zone}

Patterns of morphological and electrophoretic variation in Connecticut field crickets reveal a clear transition from $G$. firmus populations along the coast to $G$. pennsylvanicus populations in the central and northwestern parts of the state (Harrison and Arnold, 1982). Hybridization and introgression are occurring at localities in south central Connecticut. For the present study, four coastal samples (E, L, M, and W) were collected from beaches along Long Island Sound or from grassy areas and dunes adjacent to beaches. These populations have consistently low mean character index scores (fig. 1). Moreover, although there is a considerable range of I values within coastal populations, only 3 out of 76 individuals have $I>10$ (fig. 2). Populations from northwestern Connecticut $(S, U)$ have high values of $\bar{I}$ (fig. 1)

The range of character index scores is $10-18$ and only four out of 72 individuals have $I<12$ (fig. 2).

The overall pattern of variation in the hybrid zone is not one of simple monotonic clinal variation, but instead resembles a patchwork. Populations with high values of $\bar{I}$ are not restricted to sites far inland but occur adjacent to and even within the hybrid zone. Not far from the coast there are pockets of nearly "pure" G. pennsylvanicus (arrows in fig. 1) sandwiched between populations with low values of $\bar{I}$. In fact, adjacent collections may have virtually non-overlapping 

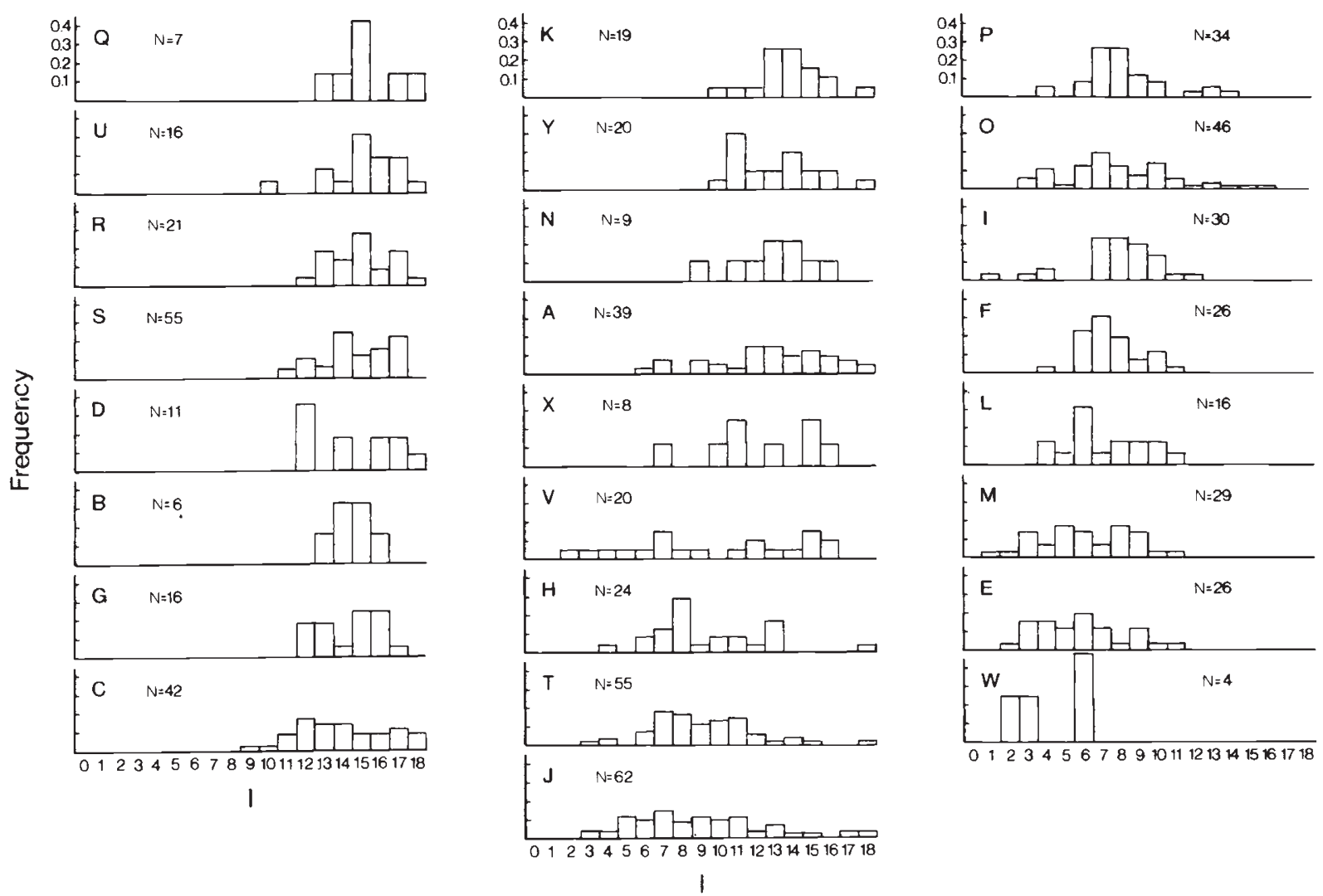

Figure 2 Distribution of individual character index scores $(I)$ for crickets from 25 localities in Connecticut. The populations are arranged in order of decreasing value of $\bar{I}$. N is the sample size for each locality.

distributions of $I$ (compare $F$ and $G$ or $P$ and $Q$ in fig. 2). These reversals in a trend of increasing $\bar{I}$ value away from the coast occur over distances of only $5-10 \mathrm{~km}$ (fig. 1). In contrast, along the Quinnipiac River Valley ( sites $O, T$, and $V$ ), I have found no "pure" G. pennsylvanicus populations, and individuals with low character index scores are found more than $50 \mathrm{~km}$ inland.

Between the extremes represented by "pure" parental populations are a series of samples with intermediate values of $\bar{I}$. These collections are not simply mixtures of the two parental types, but rather contain individuals of apparent mixed ancestry (fig. 2). By decomposing $I$ into its component parts $I_{e}$ and $I_{m}$ (or $\bar{I}$ into $\bar{I}_{e}$ and $\bar{I}_{m}$ ), we can better define the characteristics of hybrid zone populations and determine whether morphological and electrophoretic markers vary concordantly across the hybrid zone. A plot of the mean character index scores $\left(\bar{I}_{e}\right.$ against $\left.\bar{I}_{m}\right)$ (fig. 3a) clearly separates the "pure" $G$. firmus and $G$. pennsylvanicus populations. Populations that are intemediate in mean character index score appear to fall into two groups. This result is confirmed by using principal components analysis (PRINCOMP procedure in SAS). Fig. $3 \mathrm{~b}$ plots the first principal component based on electrophoretic data (frequencies of all alleles) against the first principal component based on morphology (mean values of HWLM, TEG, and OL). The similarity of Figs 3a and $3 \mathrm{~b}$ is not surprising given that (1) both character index scores and principal components represent weighted linear combinations of the electrophoretic and morphological characters and (2) both are designed to emphasise differences between the extreme forms. For the character index scores, weights given to individual characters (alleles or morphological measures) are summarised in table 2. For PC1E, the major alleles at each locus are given nearly equal weight, with $E s t^{35}, E s t^{60}, P e p-1^{110}$, and Pep- $3^{100}$ having negative loadings $(-0 \cdot 35,-0 \cdot 27,-0 \cdot 34$, and $-0 \cdot 37)$ and Est ${ }^{-10}, P e p-1^{100}, P e p-3^{70}$, and Pep- $3^{120}$ having positive loadings $(0.32,0 \cdot 38,0 \cdot 32,0 \cdot 34)$. For PC1M, the morphological measures are equally weighted (HWL, 0.57; OL, 0.59; TEG, -0.57 ). Thus, the 
a.

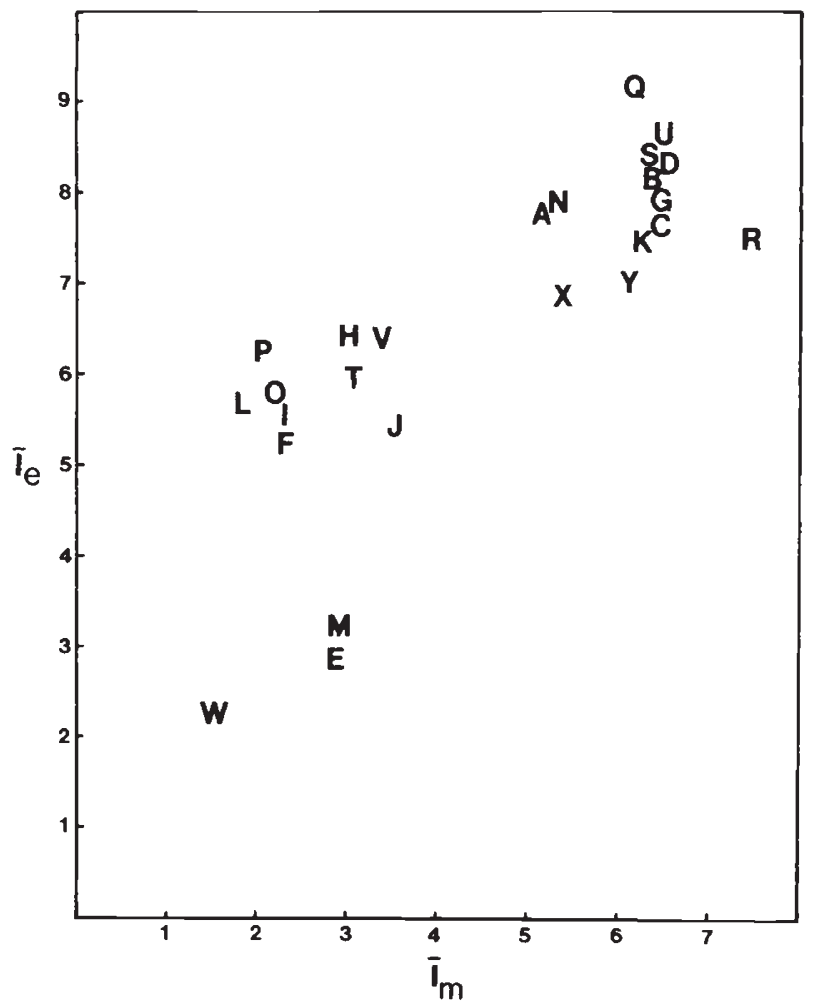

b.

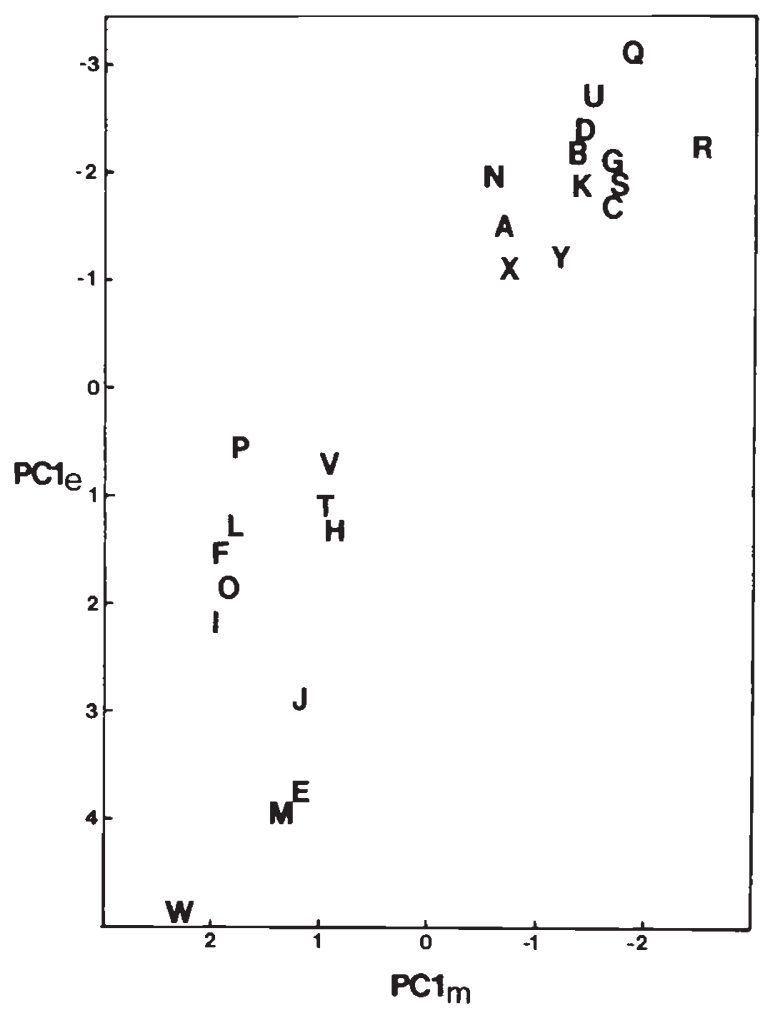

Figure 3 (a) Plot of mean character index scores for each population. $\vec{I}_{e}$ (based on genotypes at three enzyme loci) is plotted against $\vec{I}_{m}$ (based on HWLF and OL for females, HWLM and TEG for males). (b) Plot of the first principal component based on electrophoretic data (allele frequencies at three loci) against the first principal component based on morphology (mean values of HWLM, TEG, and OL).

weighting schemes for the two approaches are similar.

To summarise the morphological and electrophoretic characteristics of individual crickets within hybrid zone populations I present histograms of $I_{m}$ as a function of $I_{e}$ (fig. 4). Fig. 4a shows that such a plot clearly separates individuals from "pure" parental populations.

Populations $A, N$, and $X$ are similar to "pure" G. pennsylvanicus populations in both morphology and allozyme frequencies, but $\bar{I}_{e}$ and/or $\bar{I}_{m}$ are shifted toward lower values (fig. $3 \mathrm{a}$ ). The samples from sites $N$ and $X$ are small; their displacement away from the cluster of "pure" $G$. pennsylvanicus populations is in part a consequence of the presence in each case of a cricket that is morphologically like G. firmus (low $I_{m}$ ) but carries G. pennsylvanicus alleles (high $I_{e}$ ) (figs $4 \mathrm{~b}, \mathrm{c}$ ). The sample from site $\mathrm{A}$ is larger and contains a number of individuals with low values of $I_{m}$ and high values of $I_{e}$, as well as a single individual that might be classified as "pure" G. firmus (fig. 4d).
A second group of hybrid zone populations appears as a discrete cluster in fig. 3. Geographically, these populations are restricted to sites on or near the coast $(F, H, J, L, O, P)$ or inland along the major river valleys $(I, T, V)$ (fig. 1$)$. At some sites $(F, I, L, P)$ collections consist of a relatively homogeneous array of individuals with the morphological characteristics of G. firmus $\left(I_{m}<4\right)$ but with $G$. pennsylvanicus alleles present at higher frequency than in the "pure" G. firmus populations $\left(I_{e}>5\right)$ (fig. 2, fig. 4e-h). At other sites $(J, O, T, V)$ individuals ranging from "pure" $G$. pennsylvanicus to "pure" G. firmus coexist (fig. 2). Again, a majority of crickets in these populations are morphologically like $G$. firmus but many of these carry $G$. pennsylvanicus alleles (fig. 4i-1).

The extent to which $G$. pennsylvanicus alleles are found in crickets with G. firmus morphology can be illustrated by plotting frequencies of the Est ${ }^{-10}, P e p-1^{100}$, and Pep $-3^{100}$ alleles against $\bar{I}_{m}$ for each population (fig. 5a-c). A regression line defined by the "pure" parental populations rep- 


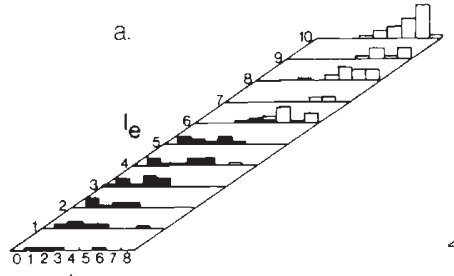

Im
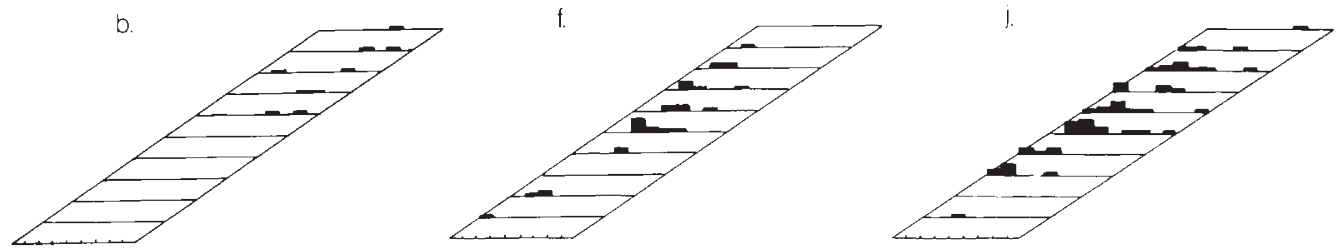
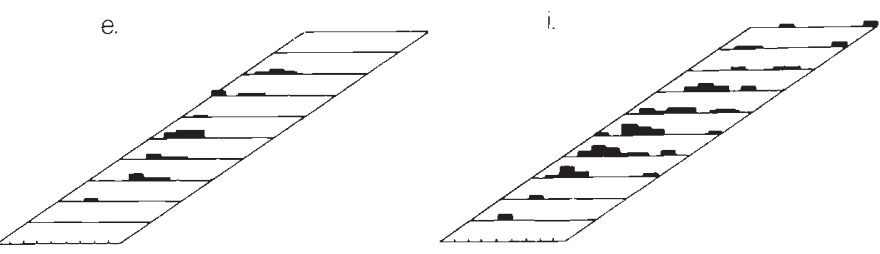
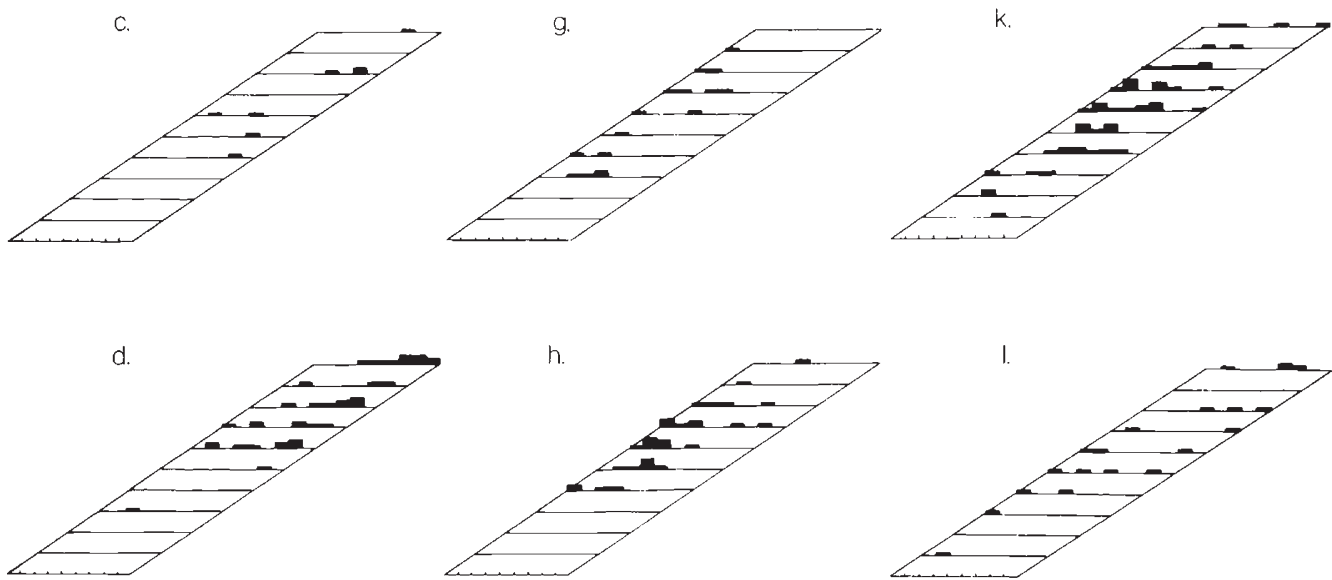

Figure 4 Distribution of individual values for $I_{e}$ and $I_{m}$. Each panel represents a series of histograms of $I_{m}$ for the range of values of $I_{e}$. The first panel (a) illustrates differences between "pure" $G$. pennsylvanicus populations $(S, U)$, shown by the open bars, and "pure" $G$. firmus populations $(E, M, W)$, sliown by the filled-in bars. The populations represented in the rest of the figure are as follows: (b) $N$, (c) $X$, (d) $A$, (e) $F$, (f) $I$, (g) $L$, (h) $P$, (i) $J$, (j) $O$, (k) $T$, (l) $V$.

resents expected values if allele frequencies and morphology vary concordantly. For each locus, allele frequencies in hybrid zone populations are shifted away from this line toward values characteristic of $G$. pennsylvanicus. The shift is most dramatic for the Est locus. The Est ${ }^{-10}$ allele occurs at high frequency in three of the four coastal populations $(E, M$, and $W$ ) and in $G$. firmus populations elsewhere in the eastern United States (Harrison and Arnold, 1982). However, in one coastal population $(L)$ and in hybrid zone populations (e.g., $F, I, O, P) E^{-10} t^{-10}$ greatly reduced in frequency, despite the fact that the great majority of crickets in these populations exhibit the morphological characteristics of G. firmus (fig. 5a). The Est ${ }^{-10}$ allele is largely replaced by $E s t^{35}$ and $E s t^{70}$, alleles characteristic of $G$. pennsylvanicus. In population $\mathrm{H}$, most crickets would be classified as $G$. firmus on the basis of morphology, but the Est ${ }^{-10}$ allele is virtually absent. For Pep-1 and Pep-3, the presence of $G$. pennsylvanicus alleles in crickets with G. firmus morphology is not as consistent nor as extensive as for Est (fig. 5b, c). Thus, in a plot of allele frequency against $\bar{I}_{m}$, Pep-1 ${ }^{100}$ frequencies in hybrid zone populations fall close to (but still consistently below) a regression line defined by the "pure" parental populations (fig. 5b).

The $E s t^{-40}$ allele exhibits a pattern distinct from any other allele or morphological character. It occurs at highest frequency in mixed/intermediate populations. This can be seen in fig. $5 \mathrm{~d}$, in which Est ${ }^{-40}$ frequency is plotted against $\bar{I}$. 
a.

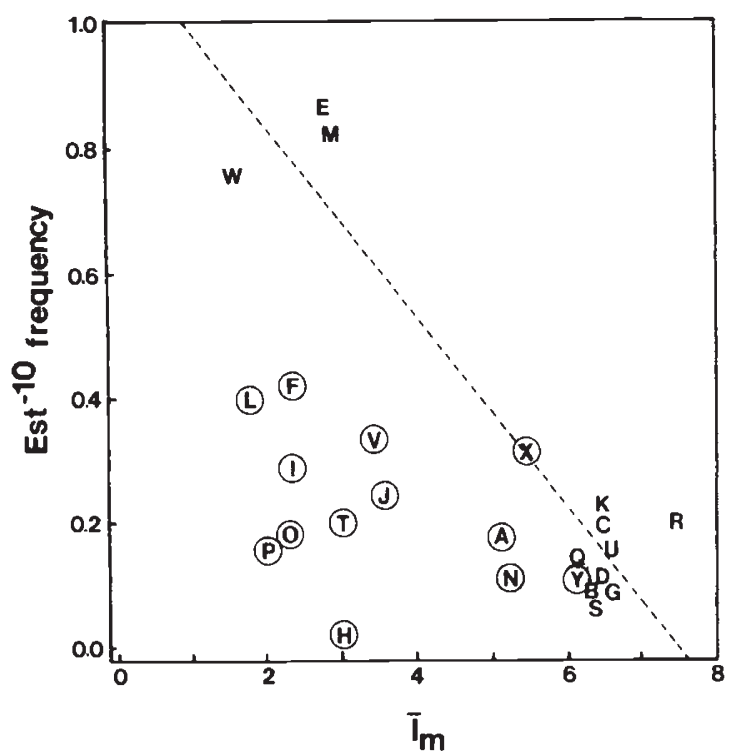

b.

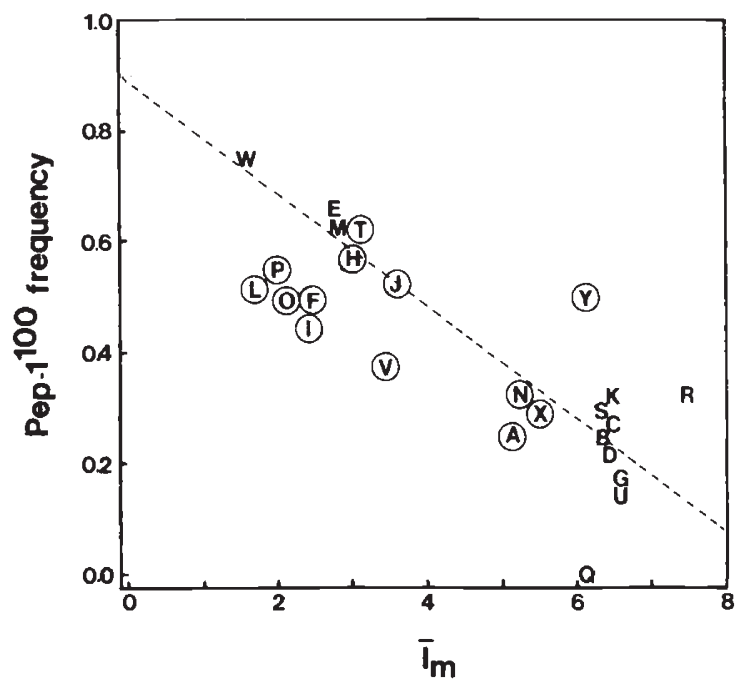

C.

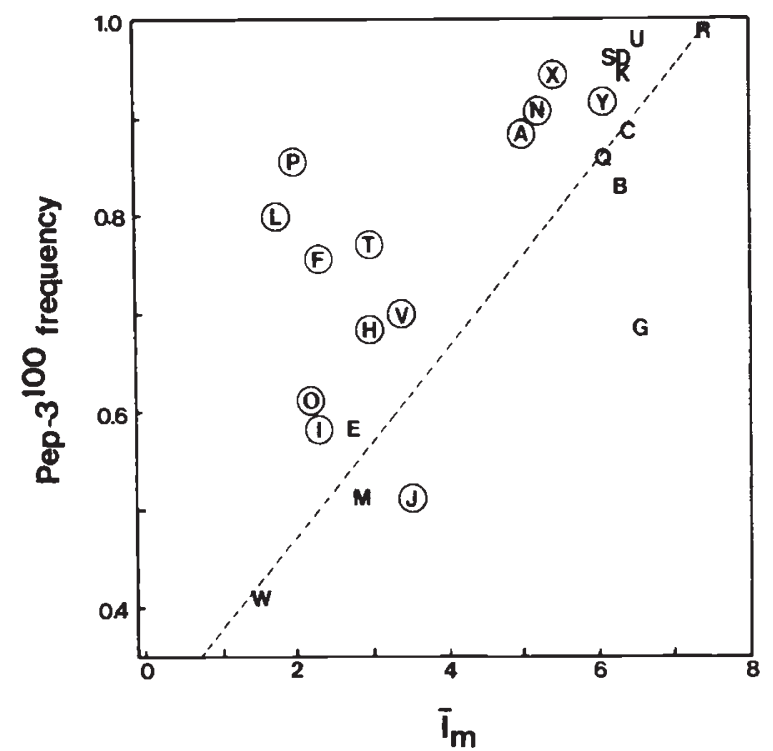

d.

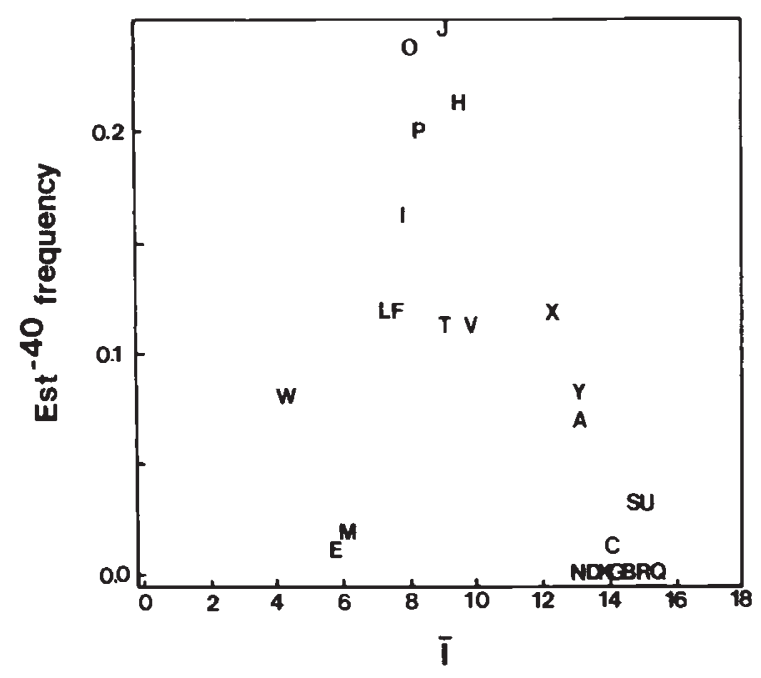

Figure 5 (a)-(c) Plots of allele frequency against $\bar{I}_{m}$ for the Est ${ }^{-10}, P e p-1^{100}$, and Pep- $3^{100}$ alleles. The dashed lines are regression lines determined by using values for populations that are considered "pure" parental (not circled). Presumed mixed/intermediate populations are circled. (d) Plot of Est frequency against the mean character index score for each population.

\section{Analysis of field-inseminated females and their offspring}

Using Est alleles as genetic markers, Harrison and Arnold (1982) concluded that there is not a clear association of electrophoretic and morphological markers in mixed/intermediate populations from the northeastern United States. Based on a large sample of females from population $\mathrm{J}$ ( HN2 of
Harrison and Arnold (1982)), they showed that $E s t^{-10}$ allele frequencies do not vary significantly among subpopulations of crickets that are morphologically distinct. The data presented above confirm and extend these earlier results for the Est locus. Moreover, in a sample of 75 females collected from population $J$ in 1980 , Est ${ }^{-10}$ frequencies are not significantly different between subpopulations with long $(>16 \mathrm{~mm})$ and short $(\leq 16 \mathrm{~mm})$ 
ovipositors $(G=1 \cdot 03, p>0 \cdot 1)$. However, Pep-3 allele frequencies are significantly different between these morphologically distinct subpopulations $(G=15.00, p<0.005)$ and therefore are useful as markers to assess the extent of positive assortative mating within this population.

The Pep $-3^{100}$ allele is fixed or occurs at high frequency $(>0.9)$ in $G$. pennsylvanicus populations. It is found at much lower frequency $(\sim 0.5)$ in G. firmus and is replaced by the Pep- $3^{120}$ and $P e p-3^{70}$ alleles. Imagine a simple mixed population with the two cricket species equally represented. A majority of females with $100 / 100$ genotypes will be $G$. pennsylvanicus. In contrast, females having the genotypes $120 / 100,100 / 70,120 / 120,120 / 70$, $70 / 70$ are likely to be $G$. firmus. With positive assortative mating, many $100 / 100$ females should produce offspring sired by males that contribute only the 100 allele (100/100 males). However, some proportion of $100 / 100$ females will be $G$. firmus. The offspring of these females must be sired by $G$. firmus males, so that these offspring on average should receive the $P e p-3^{100}$ allele only about 50 per cent of the time. Females with Pep-3 genotypes that are not $100 / 100$ should produce offspring sired by the same subpopulation of males.

Fig. 6 shows that expectations based on positive assortative mating are met in the 1980 sample from site $J$. There are consistent differences in the allelic contribution of males to the offspring of females that carry 0,1 , or 2 copies of the Pep- $3^{100}$ allele. A large fraction of $100 / 100$ females produce offspring that are predominantly or exclusively $100 / 100$ genotypes. This implies that these females have most often mated with males having the $100 / 100$ genotype. In contrast, the frequency of the 100 allele contributed by males to offspring of the remaining 100/100 females and to offspring of most females with other Pep-3 genotypes is much lower (i.e., these females have most often mated with males that have genotypes other than $100 / 100$ ). Within the group of $100 / 100$ females, those that produce a large proportion of $100 / 100$ offspring have short ovipositors $(\leq 16 \mathrm{~mm})$ and thus resemble $G$. pennsylvanicus, whereas those $100 / 100$ females that produce $10-80$ per cent $100 / 100$ offspring have long ovipositors $(>16 \mathrm{~mm})$ and resemble $G$. firmus (fig. 6). Most females that carry one or more copies of either the Pep- $3^{120}$ or the Pep- $3^{70}$ alleles have long ovipositors. Thus, there is not only an association of Pep-3 genotype and OL, but also clear evidence of assortative mating with respect to these characters. Note that assortative mating persists despite apparent "dedifferentiation" at the Est locus.
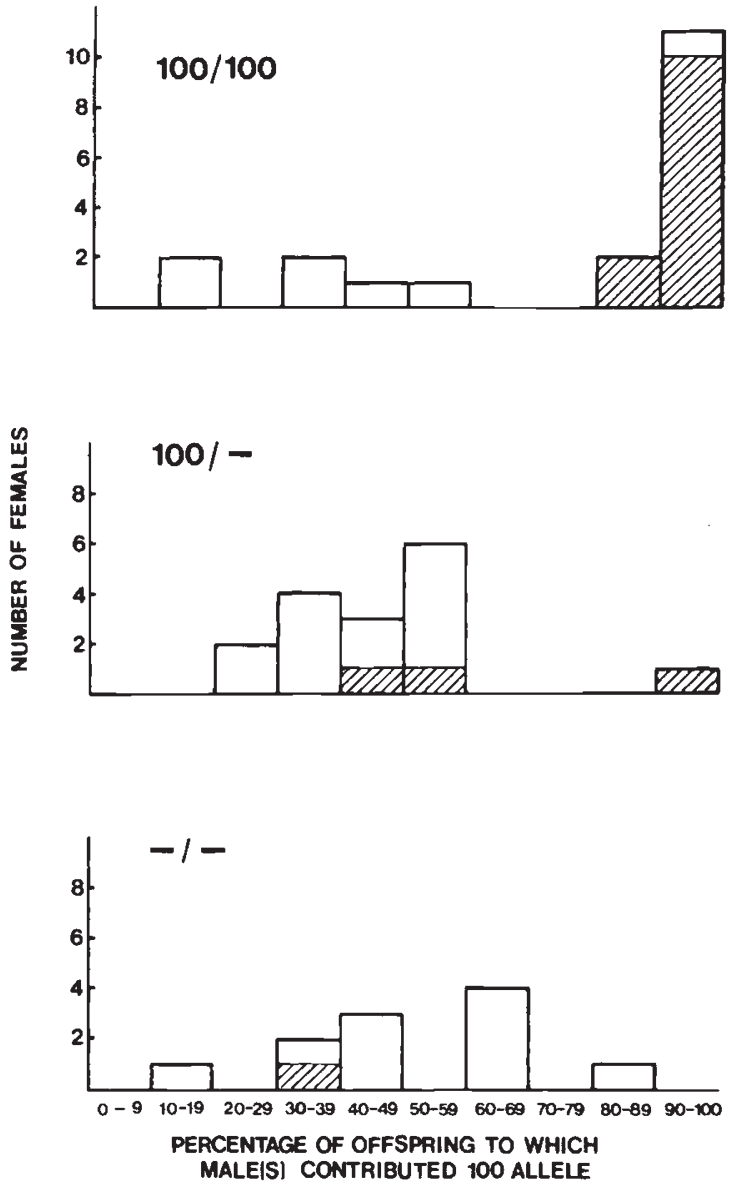

Figure 6 Histograms showing the number of females that produce offspring with the specified paternal contribution of the Pep- $3^{100}$ allele. The females were collected during the late summer and early fall in 1980 at site $J$. For purposes of comparison the females have been divided into three groups, depending on their Pep-3 genotype (those that are homozygous for the 100 allele (top panel), those that are heterozygous (middle panel), and those that lack the 100 allele (bottom panel)). Each group is further subdivided on the basis of ovipositor length. The hatched bars (or portions thereof) represent females with $\mathrm{OL} \leq 16 \mathrm{~mm}$. Open bars are females with $\mathrm{OL} \leq 16 \mathrm{~mm}$

\section{DISCUSSION}

\section{The structure of the hybrid zone}

In most theoretical treatments, hybrid zones are portrayed as monotonic clines maintained by a balance between gene flow and selection. The selection component of these models involves either the reduced fitness of hybrids or spatial variation in selection pressures. The patterns of variation across the Gryllus pennsylvanicus-G. firmus hybrid zone do not conform to a simple 
model of monotonic clinal variation. Although, at low resolution, the pattern of variation involves a transition from G. firmus characters along the coast to $G$. pennsylvanicus characters inland, the hybrid zone itself appears to be a mosaic of populations. Any explanation for the origin and maintenance of the hybrid zone must account for this pattern of variation.

The pattern of the mosaic may be determined simply by the recent history of these cricket species in Connecticut, e.g., by colonisation and extinction events during secondary contact of already differentiated forms. Although selection may play a role in this process, it need not be invoked to explain the pattern of variation. Available habitat for the two species has no doubt greatly increased over the past several hundred generations. Species that may have once been restricted to beaches and sandy river deposits ( $G$. firmus) or small woods openings and steep rocky slopes (G. pennyslvanicus) are now much more widely distributed.

However, an alternative explanation for the mosaic is that the distribution of character states for each character reflects current selection pressures imposed by the environment. That is, patterns of variation across the zone may reflect an underlying habitat and/or resource mosaic, with some habitats/resources favouring one species (or combination of character states) and other habitats/resources favouring the second species (or a different combination of character states).

If the environment is a mosaic of selection pressures, how will an organism respond? For a single-locus model, Slatkin (1973) considered the effects of a "pocket" in a one-dimensional environment. He defined a "pocket" as a region in which one homozygote is favored surrounded by regions in which the other homozygote has a selective advantage. If the size of the pocket is greater than the "characteristic length" $\left(l_{c}\right)$ for spatial variation at the locus under consideration, then variation at that locus will reflect the presence of the pocket. The characteristic length is defined as $l / \sqrt{s}$, where $l$ is the average dispersal distance and $s$ is a measure of the strength of selection. In Connecticut, both $G$. pennsylvanicus and $G$. firmus are flightless; hence individual dispersal distances are not likely to be very great (perhaps of the order of tens or hundreds of metres). Thus, the characteristic length may be small even if selection coefficients for the characters under consideration are not large (e.g., if $l=100 \mathrm{~m}$ and $s=0.0001$, then $l_{c}=10 \mathrm{~km}$, approximately the size of observed "pockets" within the cricket hybrid zone). In fact, the spatial scale of patches (or the "grain" size
(Levins, 1968)) may be smaller than indicated by the data presented here (requiring larger selection coefficients if selection is to explain the pattern of variation). Obviously, the sampling scheme used in this study would not detect spatial variation over distances less than the distances between collecting sites. Detailed mapping of this hybrid zone suggests that pockets of local abundance of the two species actually occur on a scale much finer than represented here (D. M. Rand, personal communication). Preliminary data also suggest that the mosaic nature of this hybrid zone may result from (or is at least correlated with) the patchy nature of soil types in south-central Connecticut. Crickets with many of the characteristics of G. firmus (the "beach cricket") appear to be restricted to areas of sandy soil (i.e., near the coast and along river valleys), whereas $G$. pennsylvanicus is found principally on loam. Selection for long ovipositor and/or light coloured tegmina in habitats with sandy soils has been invoked to explain patterns of morphological variation in crickets at other localities (Lutz, 1908; Alexander, 1968).

A third possible explanation for the pattern of variation is that the environment is a mosaic of different patch types (as required by the selection model), but distributions are a result of crickets selecting habitats rather than habitats selecting crickets. The literature on field crickets suggests that each species tends to be associated with certain habitat types (e.g., woods versus pastures, fields, and roadsides). Experimental evidence (Howard and Harrison, 1984) indicates that crickets in the genus Allonemobius choose between wet and dry areas within the same pasture. Thus, habitat selection certainly could be part of the repertoire of the field crickets involved in the hybrid zone interaction.

\section{Consequences of a mosaic hybrid zone}

The notion that hybrid zones are mosaics rather than simple clines has a number of consequences for thinking about the dynamics of these zones. First, it emphasises that the differentiated forms (species) involved in hybrid zones often interact in discrete local populations and that a single hybrid zone will therefore involve many interactions between these forms. Since interactions in different parts of the zone are presumably independent (especially for organisms of low vagility), the outcome may vary from site to site. Deterministic population genetic models of hybrid zone interactions (e.g., Paterson, 1978) suggest that if hybrids are less fit than parentals the rarer parental type 
(or the rarer alleles at each locus contributing to hybrid unfitness) will be eliminated. Paterson (1978) uses this model to argue that extinction, not speciation, is the likely outcome of such a hybrid zone interaction. But in a mosaic hybrid zone global extinction of either form will be less likely, if proportions of the two forms vary from site to site.

If hybrids have reduced fitness, it is difficult for one species to invade a continuous population of the second species, since the recent colonist will almost always be rare compared with the established resident. Placing hybrid zones in the context of a patchy environment raises the possibility of "empty patches". Particularly in species that occupy disturbed habitats (and G. pennsylvanicus and G. firmus are such species), there is likely to be considerable turnover of suitable habitat. If a new patch becomes available within the range of species 1 , invasion by species 2 can occur without incurring any "cost of hybridisation". Unlike models in which species are continuously distributed, a patch or mosaic model suggests that there is a premium on dispersal, since first "possession" of a patch may be very important. This assumes that selection against hybrids is a more important force than differential success of alleles of the two species in patches of different types.

\section{Differential introgression and the semi- permeable nature of species boundaries}

Patterns of variation in morphology and allele frequencies are not concordant across the hybrid zone. Indeed most hybrid zone populations include a large proportion of individuals that look like G. firmus but carry G. pennsylvanicus alleles, particularly at the Est locus (figs. 4e-1). The simplest explanation for these observations is that there has been differential introgression of $G$. pennsylvanicus alleles into $G$. firmus, with alleles at the Est locus introgressing to a greater extent than alleles at the other two loci. Despite apparent introgression at the allozyme loci, morphological integrity appears to be maintained. $\vec{I}_{m}$ for samples from $F, I, L, O$, and $P$ is, in fact, lower than for samples from two of the three "pure" $G$. firmus populations.

Evidence for introgression of $G$. firmus alleles into crickets with $G$. pennsylvanicus morphology is far less convincing. Within and adjacent to the hybrid zone, populations of crickets that are $G$. pennsylvanicus in morphology sometimes have higher frequencies of G. firmus alleles than "pure"
G. pennsylvanicus populations (e.g., populations $C, K, R$, and $X$ for Est (fig. 5a) or populations $B$, $G$ and $Q$ for Pep-3 (fig. 5c)). However, the pattern is not consistent and the frequency differences might represent intraspecific variation and/or sampling variance. The apparent asymmetry in extent of introgression is similar to patterns observed in other hybrid zones. A distinct asymmetry in introgression has been documented for allozymes in the Mus musculus-Mus domesticus hybrid zone in Europe (Hunt and Selander, 1973). Barton and Hewitt (1981) found this asymmetry "puzzling". They suggested that recent movement of the zone could produce the pattern, but they ruled out this possibility in the case of the house mouse interaction. In the cricket hybrid zone recent movement of both species into newly available habitat, combined with selective elimination of alleles, could have produced the observed pattern. G. pennsylvanicus alleles at Est (and to a lesser extent, Pep-1 and Pep-3) are retained, whereas $G$. pennsylvanicus alleles at loci controlling certain aspects of morphology are rapidly eliminated.

A lack of concordance between allozyme variation and morphological variation has also been observed in other hybrid zones (e.g., McDonnell et al., 1978; Patton et al., 1979). In a hybrid zone between two "races" of pocket gophers, Patton et al. (1979) observed introgression of alleles at allozyme loci beyond the hybrid zone defined on morphologic grounds. In their study, morphology correlated well with an ecological or habitat gradient, providing indirect evidence that "there is a stronger interaction between an individual's morphology... and the microhabitat in which it occurs than between either of these features and its genotype [genotype means allozyme alleles and chromosomal complement]". The data from the cricket hybrid zone are consistent with this conclusion.

The population of crickets at site $J$ includes both "pure" parental types and individuals of mixed ancestry (principally individuals with $G$. firmus morphology and $G$. pennsylvanicus alleles). Despite evidence for past introgression at the Est locus, there is strong positive assortative mating at this site. Females with short ovipositors and Pep$3^{100 / 100}$ genotypes almost always produce offspring sired by $P e p-3^{100 / 100}$ males. Female field crickets are often multiply-inseminated. Offspring produced in the laboratory by a field-inseminated female mostly reflect the contribution of the last male with which she mated. However, some contribution of sperm from earlier matings is expected (Harrison, unpublished data). Therefore, these 
data suggest that at site $J G$. pennsylvanicus females mate primarily or exclusively with conspecific males.

Although the two taxa have become dedifferentiated at the Est locus, they apparently retain morphological distinctness and at least some of the ecological or behavioural differences responsible for mate selection (see below). In this case (and perhaps for many pairs of hybridising taxa), the species boundary is best viewed as "semipermeable", the permeability varying with the genetic marker used (see Barton, 1979a; Barton and Hewitt, 1981, for similar ideas expressed in different terms). Genetic exchange between species can occur at some loci without further compromising the integrity of the species boundary itself. (Since some hybridisation is occurring, the boundary must already be "compromised" to some extent.) Recent data on introgression of mitochondrial DNA has focused attention on this phenomenon for a maternally inherited marker that is unlinked to any nuclear genes (Powell, 1983; Ferris et al., 1983; Barton and Jones, 1983).

\section{Another example of the "rare allele phenomenon"}

The pattern of variation for the $E s t^{-40}$ allele is distinctive. The allele always occurs at low frequency outside the hybrid zone, but is found at relatively high frequency in most of the mixed/intermediate populations. Other hybrid zone studies have documented similar phenomena (the "rare allele phenomenon"- - see Sage and Selander, 1979). One explanation for this pattern is that the allele is of hybrid origin, perhaps having arisen via intragenic recombination in an individual of mixed ancestry (see Watt, 1972). Of course, other explanations could also be invoked-e.g. it could be argued that this allele is only favoured in those habitats occupied by crickets of mixed ancestry.

\section{What is the basis of assortative mating within the hybrid zone?}

Previously I have shown that in the laboratory $G$. pennsylvanicus females can produce fertile offspring sired by males of either species, but $G$. firmus females produce offspring only when mated with conspecific males (Harrison, 1983). This asymmetric post-mating barrier cannot explain why $G$. pennyslvanicus females in mixed populations produce offspring sired primarily by $G$. pennsylvanicus males. Nor can the evidence for assortative mating be explained by temporal isola- tion, since adults of both species appear synchronously at site $J$ (Harrison, 1985). The two most likely explanations for positive assortative mating are (1) habitat isolation and/or (2) behavioral isolation involving mate choice by female crickets. I have already suggested that the two cricket species occupy distinct habitats (or soil types). If this association extends to a very fine spatial scale, both habitat types may occur at site $J$, and crickets could be spatially (ecologically) separated.

The traditional view of mate choice in crickets is that male crickets produce a species-specific calling song that attracts conspecific females (Alexander, 1962). Alexander (1957) reported that the songs of $G$. firmus and $G$. pennsylvanicus are similar, but can be distinguished by differences in pulse repetition rate. Analysis of songs of males from allopatric populations in Connecticut (sites $E$ and $S$ ) reveal only very small differences in mean pulse repetition rate, with some overlap between the two species (Harrison, unpublished). Thus, song differences are not necessarily an obvious basis for mate choice in this system. However, experiments in which $G$. pennsylvanicus females are caged in the laboratory with paired males of $G$. firmus and $G$. pennsylvanicus (from sites $E$ and $S$, respectively) result in females producing offspring sired primarily or exclusively by conspecific males (Harrison, unpublished). Thus, there is reason to believe that assortative mating has a behavioural component.

Evidence for positive assortative mating in Connecticut contrasts with observations of interactions between the same pair of species in Virginia. Along the Blue Ridge, adults of G. firmus and $G$. pennsylvanicus are temporally isolated (Harrison, 1985). But during the period in late summer when adults of the two species do occur together, there is no evidence of positive assortative mating (Harrison 1985). In Connecticut, where temporal isolation is not a factor, there appears to be strong positive assortative mating. It is tempting to argue that selection against hybridisation has led to behavioral isolation at sites where other barriers to gene exchange are not operating. The existence of behavioural barriers between crickets from "pure" allopatric populations ( $E$ and $S$ ), however, suggests that the differences responsible for these barriers may have arisen prior to formation of the hybrid zone or at least independent of it. Certainly, a detailed analysis of the "cost of hybridisation" in this system must precede any detailed discussion of whether reinforcement has played a role in the origin of barriers to gene exchange. 
Acknowledgements I thank Susan Marafino and Janice Chappell for expert technical assistance, John Kochmer and David Rand for many critical discussions of hybrid zones and their significance, and an anonymous reviewer for several helpful comments. This research was supported by NSF grants BSR8111603 and BSR-8407474.

\section{REFERENCES}

ALEXANDER, R. D. 1957. The taxonomy of the field crickets of the eastern United States (Orthoptera: Gryllidae: Acheta). Ann. Entomol. Soc. Amer., 50, 584-602.

ALEXANDER, R. D. 1962. Evolutionary change in cricket acoustical communication. Evolution, 16, 443-467.

ALEXANDER, R. D. 1968. Life cycle origins, speciation, and related phenomena in crickets. Quart. Rev. Biol., 43, 1-41.

AYALA, F. J., TRACEY, M. L., HEDGECOCK, D. AND RICHMOND, R. C. 1974. Genetic differentiation during the speciation process in Drosophila. Evolution, 28, 576-592.

BARTON, N. H. 1979a. Gene flow past a cline. Heredity, 43, 333-339.

BARTON, N. H. 1979b. The dynamics of hybrid zones. Heredity, $43,341-359$.

BARTON, N. H. AND HEWITT, G. M. 1981. Hybrid zones and speciation. In Evolution and Speciation, W. R. Atchley and D. S. Woodruff (eds). Cambridge University Press.

BARTON, N. H. AND JONES, J. S. 1983. Mitochondrial DNA: new clues about evolution. Nature, 306, 317-318.

BIGELOW, R. S. 1965. Hybrid zones and reproductive isolation. Evolution, 19, 449-458.

CROSBY, J. L. 1970. The evolution of genetic discontinuity: computer models of the selection of barriers to interbreed. ing between subspecies. Heredity, 25, 253-297.

DobZhANSKY, T. 1970. Genetics of the Evolutionary Process. Columbia University Press.

ENDLER, J. A. 1977. Geographic Variation, Speciation and Clines. Princeton University Press.

ENDLER, J. A. 1983. Testing causal hypotheses of geographical variation. In Numerical Taxonomy, J. Felsenstein (ed.). Springer-Verlag.

FERRIS, S. D., SAGE, R. D., HUANG, C. M., NIELSEN, J. T. RITTE, U, AND WILSON, A. C. 1983. Flow of mitochondrial DNA across a species boundary. Proc. Natl. Acad. Sci. USA, 80, 2290-2294.

HALL, W. P. AND SELANDER, R. K. 1973. Hybridization in karyotypically differentiated populations of the Sceloporus grammicus complex (Iguanidae). Evolution, 27, 226-242.

HARRISON, R. G. 1979. Speciation in North American field crickets: evidence from electrophoretic comparisons. Evolution, 33, 1009-1023.

HARRISON, R. G. 1983. Barriers to gene exchange between closely related cricket species. I. Laboratory hybridization studies. Evolution, 37, 245-251.

HARRISON, R. G. 1985. Barriers to gene exchange between closely related cricket species. II. Life cycle variation and temporal isolation. Evolution, 39, 244-259.

HARRISON, R. G. AND ARNOL.D, J. 1982. A narrow hybrid zone between closely related cricket species. Evolution, 36, 535552.

HOWARD, D. J. AND HARRISON, R. G. 1984. Habitat segregation in ground crickets: the role of interspecific competition and habitat selection. Ecology, 65, 69-76.
HUNT, W. G. AND SEL.ANDER, R. K. 1973. Biochemical genetics of hybridisation in European house mice. Heredity, 31, 11-33.

JACKSON, J. F. 1973. The phenetics and ecology of a narrow hybrid zone. Evolution, 27, 58-68.

LEVINS, R. 1968. Evolution in Changing Environments. Princeton University Press.

LUTZ, F. E. 1908. The variation and correlation of certain taxonomic characters of Gryllus. Carnegie Inst. Washington Publ. No. 101, 3-63.

MAYR, E. 1942. Systematics and the Origin of Species. Columbia University Press.

MAYR, E. 1963. Animal Species and Evolution. Belknap Press, Harvard.

MCDONNELL, L. J., GARTSIDE, D. F. AND LITTLEJOHN, M. J. 1978. Analysis of a narrow hybrid zone between two species of Pseudophryne (Anura: Leptodactylidae) in south-eastern Australia. Evolution, 32, 69-81.

MOORE, J. A. 1957. An embryologist's view of the species concept. In The Species Problem, ed. E. Mayr. American Association for the Advancement of Science.

MOORE, W. S. 1977. An evaluation of narrow hybrid zones in vertebrates. Quart. Rev. Biol., 52, 263-277.

NEI, M. 1972. Genetic distance between populations. Amer. Natur., 106, 283-292.

NICKLE, D. A. AND WALKER, T. J. 1974. A morphological key to the field crickets of southeastern United States (Orthoptera: Gryllidae: Gryllus). Florida Entomol, 56, 8-12.

PATERSON, H. E. H. 1978. More evidence against speciation by reinforcement. S. Afr. J. Sci., 74, 369-371.

PATERSON, H. E. H. 1982. Perspective on speciation by reinforcement. S. Afr. J. Sci., 78, 53-57.

PATTON, J. L., HAFNER, J. C., HAFNER, M. S., AND SMITH, M. F. 1979. Hybrid zones in Thomomys bottae pocket gophers: genetic, phenetic, and ecologic concordance patterns. Evolution, 33, 860-876.

POWELL, J. R. 1983. Interspecific cytoplasmic gene flow in the absence of nuclear gene flow: evidence from Drosophila. Proc. Natl. Acad. Sci. USA, 80, 492-495.

REMINGTON, C. L. 1968. Suture-zones of hybrid interaction between recently joined biotas. Evol. Biol., 3, 321-428.

SAGE, R. D. AND SELANDER, R. K. 1979. Hybridization between species of the Rana pipiens complex in central Texas. Evolution, 33, 1069-1088.

SHORT, L. L. 1972. Hybridization, taxonomy, and avian evolution. Ann. Missouri Bot. Garden, 59, 447-453.

SLATKIN, M. 1973. Gene flow and selection in a cline. Genetics, $75,733-756$.

SLATKIN, M. 1975. Gene flow and selection in a two locus system. Genetics, 81, 787-802.

TEMPLETON, A. R. 1981. Mechanisms of speciation-a population genetic approach. Ann. Rev. Ecol. Syst., 12, 23-48.

THORPE, R. S. 1984. Primary and secondary transition zones and population differentiation: a phylogenetic analysis of range expansion. Evolution, 38, 233-243.

WALKER, T. J. 1974. Character displacement and acoustic insects. Amer. Zool., 14, 1137-1150.

WATT, W. B. 1972. Intragenic recombination as a source of population genetic variability. Amer. Natur., 106, 737-753.

WEST-EBERHARD, M. J. 1983. Sexual selection, social competition and speciation. Quart. Rev. Biol., 58, 155-183.

WILSON, E. O. 1965. The challenge from related species. In The Genetics of Colonizing Species, H. G. Baker and G. L. Stebbins (eds) Academic Press. 